Reductive Perturbation Method in Nonlinear Wave Propagation .

Formation and Developement of Shock. Waves in a Nonuniform Duct

\author{
浅 野功䇏 (名火理学部) \\ (昭和 43 年 11 月26日受理)
}

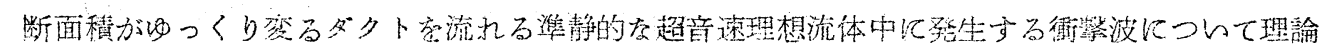

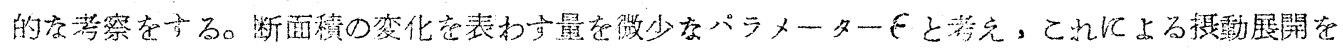
行䏠的の 2 次索で芳充た。

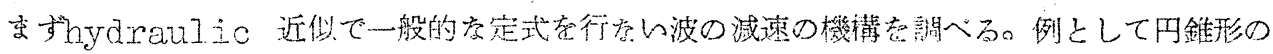
convergence 持つダクトの場合がくわしく調べられconvergenceの入口で流涑が時

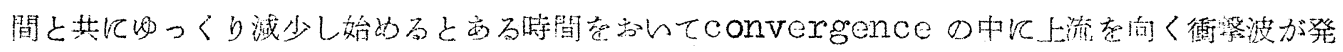

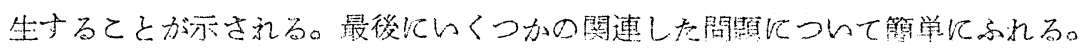

\title{
\&1. 序
}

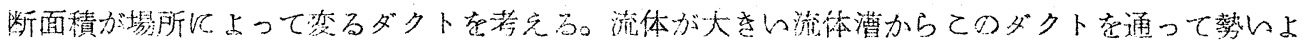

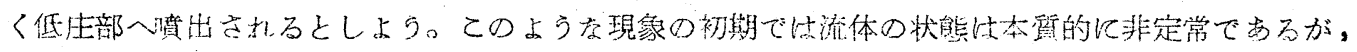

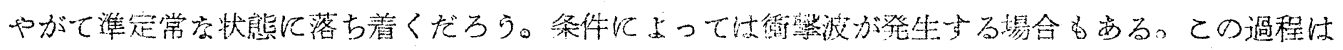
現象論的には次の3つの段階に分けると亡ができる。

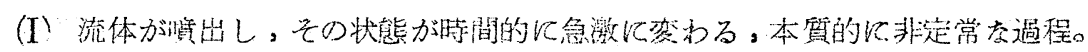

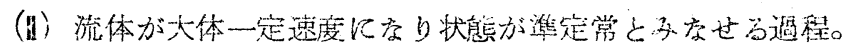

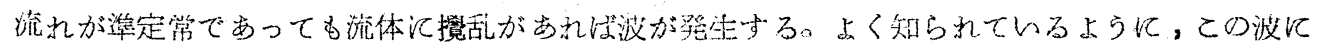

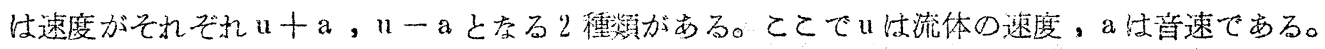

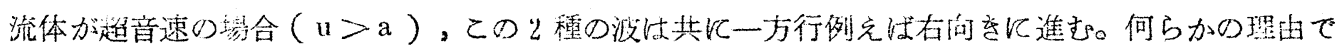

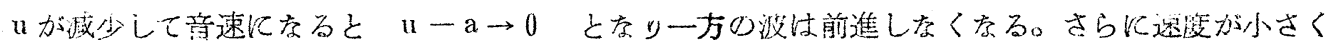




\section{浅 野}

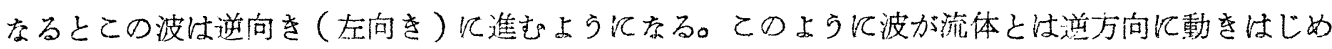
ると後に続く同様们波と衙突しで衝慗波が発生寸る。超音速で啭出した流体の速さがダクトの入口 でだんだん減少してゅく場合奆考えよう。ての場合 3 番目の段階は

(得) 衝繁波が発生しそれが上流へ伝わる過程

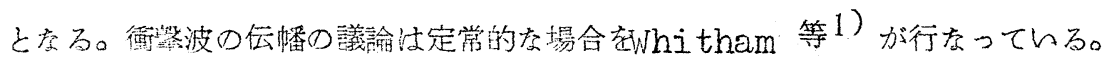

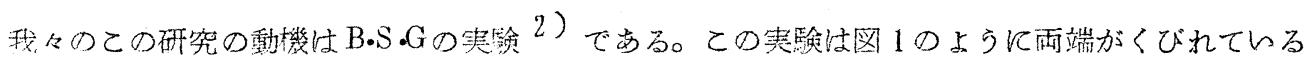
磁場の中へブラズマを德出させる過程を含む。この過程でプラズマは次の3つ状態を経る。(1) $\theta$ ピ ンチBから磁場チャンネルSの中への澎張。乙のとをプラズマ流は一般に超音速である。(2)Sでの

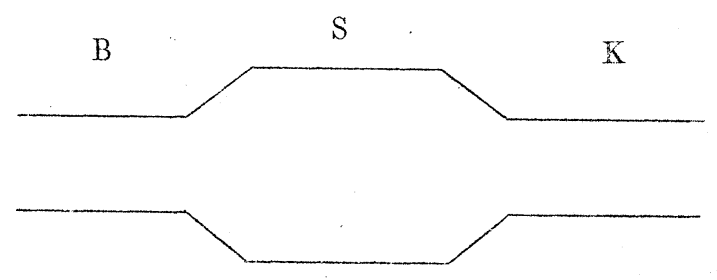

図 1 プラズマ加熱の庆的の㺊場

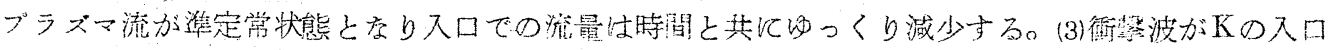

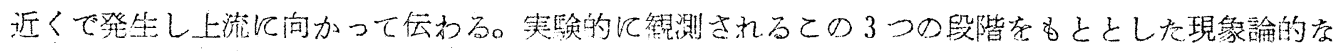

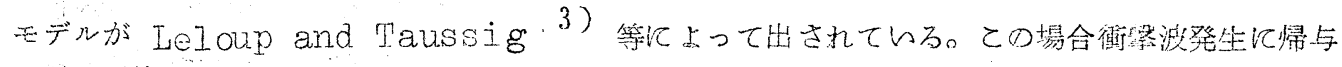
するものはBでの流速の減少とSからKへかけての磁䭪の收束である。

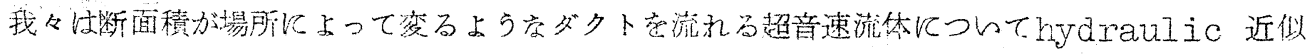

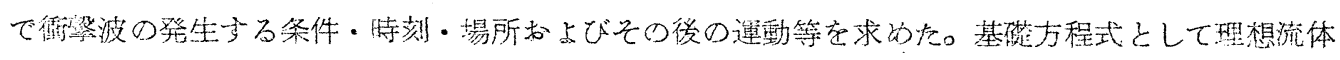

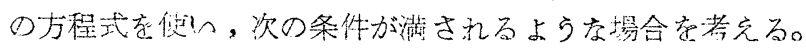

(1) 波の振巾（一様㞭状態からのずれ）江有限であるが小さん。

(2) ダクトの断面種㤌空阔的に扐っくり変る。

(3) 流体の状態沬準定常である。

この問題大披ら為に、ダクトの断面積の变化を表わす量夺小さなパラメーターをと考え, 種々の量 
Reductive Perturbation Method in Nonlinear Wave Propagation III. Formation and Developement of Shock Waves in a Non-uniform Duct

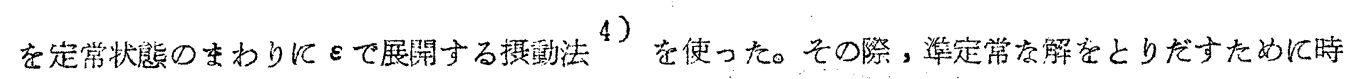

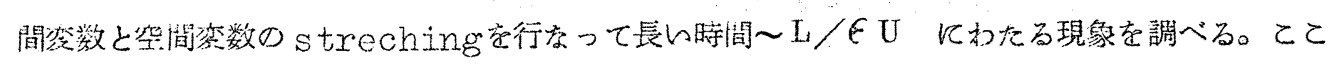
でUは衙路波の速さ，上は系の大きざである。

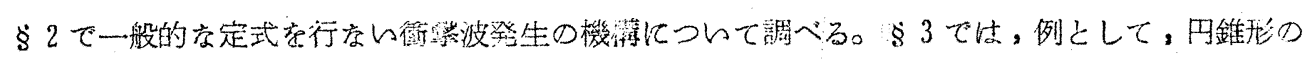

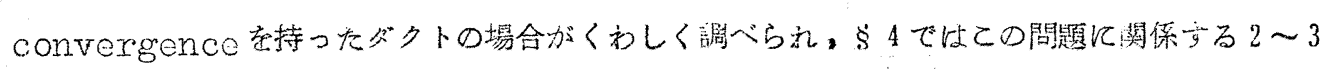
の問䟎にふれる。

\section{$\$ 2$ 一般豴}

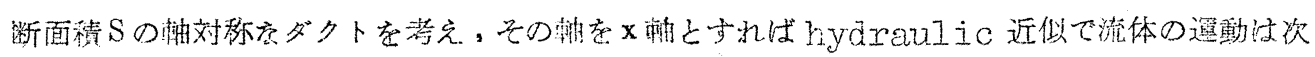
の式で表わさ记る。

$$
\begin{aligned}
& \frac{\partial \rho}{\partial \mathrm{t}}+\frac{\partial \rho \mathrm{u}}{\partial \mathrm{x}}+\frac{1}{\mathrm{~s}} \frac{\partial \mathrm{s}}{\partial \mathrm{x}} \rho \mathrm{u}=0 \\
& \rho \frac{\partial \mathrm{u}}{\partial \mathrm{t}}+\rho \mathrm{u} \frac{\partial \mathrm{u}}{\partial \mathrm{x}}+\frac{\partial \mathrm{p}}{\partial \mathrm{x}}=0 \\
& \frac{\partial \mathrm{w}}{\partial \mathrm{t}}+\frac{\partial \mathrm{q}}{\partial \mathrm{x}}+\frac{1}{\mathrm{~s}} \frac{\partial \mathrm{s}}{\partial \mathrm{x}} \mathrm{q}=0
\end{aligned}
$$

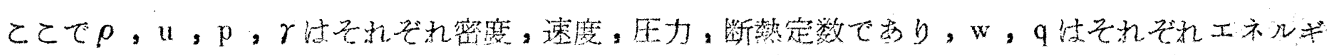
一とエネルギー流であり次式で与えられる。

$$
\left.\begin{array}{l}
w=\frac{1}{2} \rho u^{2}+\frac{1}{r-1} p \\
q=(w+p) u
\end{array}\right\}
$$

h⿱

$$
\boldsymbol{U}=\left(\begin{array}{l}
\rho \\
\mathrm{u} \\
\rho
\end{array}\right)
$$

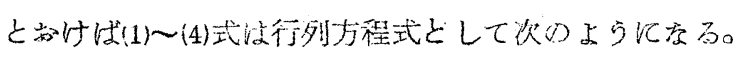

$$
\Gamma U_{t}+A U_{x}+\frac{1}{s} \frac{\partial s}{\partial x} B=0
$$


ここでU ${ }_{t}, U_{x}$ はそれぞれ $t$ xでUを偏微分したものであり

$$
\begin{aligned}
& \Gamma=\left(\begin{array}{ccc}
1 & 0 & 0 \\
0 & \rho & 0 \\
\frac{1}{2} \rho u^{2} & \rho u & \frac{1}{\gamma-1}
\end{array}\right) \\
& A=\left(\begin{array}{ccc}
u & \rho & 0 \\
0 & \rho u & 1 \\
\frac{1}{2} u^{3} \frac{\gamma}{\gamma-1} p+\frac{3}{2} \rho u^{2} \frac{\gamma}{\gamma-1} p u
\end{array}\right)
\end{aligned}
$$

及び

$$
B=\left(\begin{array}{c}
\rho u \\
0 \\
\frac{1}{2} \rho u^{3}+\frac{r}{\gamma-1} p u
\end{array}\right)
$$

である。

さて，ダクトの断面積 $\mathrm{s}$ が一定であれば，流れは一様になり，てのときの状態は定常的であると

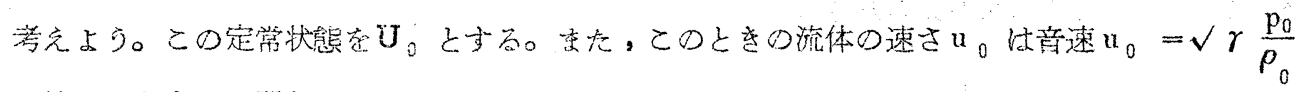
飞等しいとする。即ち

$$
U_{0}=\left(\begin{array}{l}
\rho_{0} \\
a_{0} \\
p_{0}
\end{array}\right) \text {. }
$$

次の(i)(ii)を仮定する。

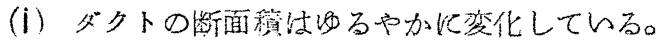

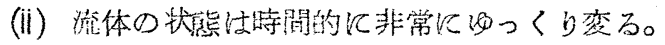

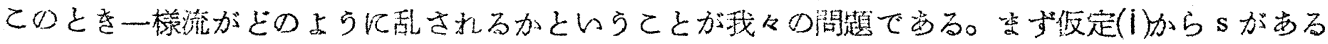
小さいパラメーターヒについて 
Reductive Perturbation Method in Nonlinear Wave Propagation III. Formation and Developement of Shock Waves in a Non-uniform Duct

$$
\mathrm{s}=\mathrm{s}_{0}+\epsilon^{2} \mathrm{~s}_{2}+\epsilon^{3} \mathrm{~s}_{3}+\cdots
$$

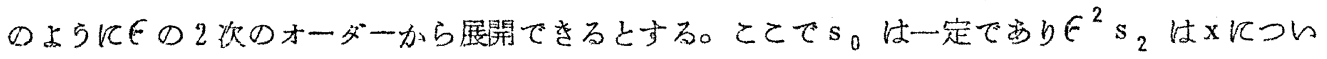

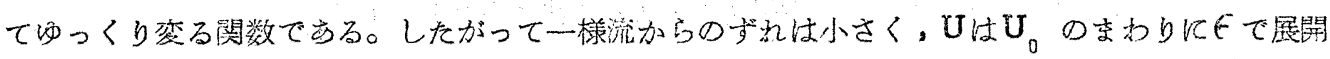
でを

$$
\mathrm{U}=\mathrm{U}_{0}+\epsilon \mathrm{U}_{1}+\epsilon^{2} \mathrm{U}_{2}+\cdots
$$

で与充られ，それに㐫じて他の量も

$$
\left.\begin{array}{l}
\Gamma=\Gamma_{0}+E \Gamma_{1}+\cdots \\
A=A_{0}+E A_{1}+\cdots \\
B=B_{0}+\epsilon B_{1}+\cdots
\end{array}\right\}
$$

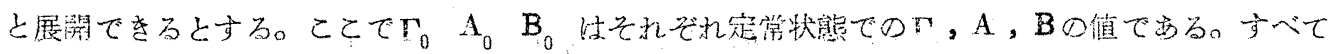

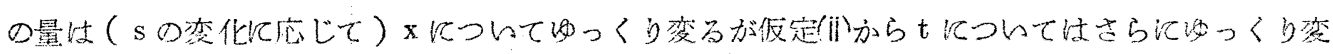

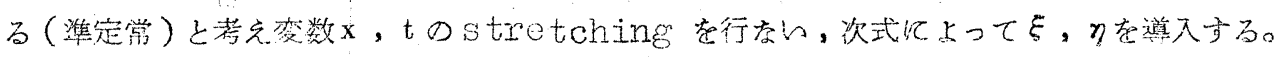

$$
\left.\begin{array}{l}
\eta=\epsilon^{2}, \\
\xi=\epsilon \mathrm{x},
\end{array}\right\}
$$

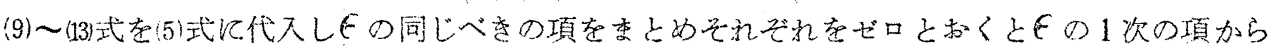

$$
A_{0} U_{1 \xi}=0
$$

をた，2次の項加ら

$$
\mathrm{U}_{1 \eta}+\mathrm{A}_{0} \mathrm{U}_{2 \xi}+\mathrm{A}_{1} \mathrm{U}_{1 \xi}+\frac{\mathrm{s}_{2 \xi}}{\mathrm{s}_{0}} \mathrm{~B}_{0}=0
$$

を得る。 $u_{0}=a_{0}$ に対してはdet $A_{0}=0$ であるから $A_{0}$ の右固有ベクトルを、（固有値ゼロ） とすれ壮佁式からU，の一般解注

$$
\mathrm{U}_{1}=\mathbf{x} \overline{\mathrm{u}}_{1}+\mathrm{V}(\eta)
$$

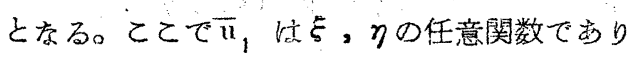




$$
V(\eta)=\left(\begin{array}{ll}
\mathrm{v}_{1} & (\eta) \\
\mathrm{v}_{2} & (\eta) \\
\mathrm{v}_{3}(\eta)
\end{array}\right)
$$

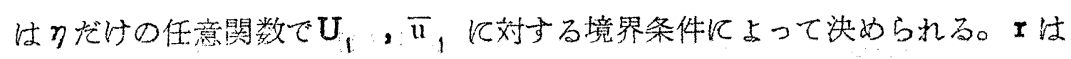

$$
r=\left(\begin{array}{cc}
-\rho_{0} \\
a_{0} \\
-\rho_{0} & u_{0}^{2}
\end{array}\right)
$$

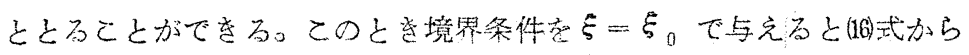

$$
\mathrm{V}(\eta)=\mathrm{U}_{1}\left(\xi_{0}, \eta\right)-\mathrm{I} \bar{u}_{1}\left(\xi_{0}, \eta\right)
$$

亲をは

$$
\begin{aligned}
& \mathrm{v}_{1}(\eta)=\rho_{0} \overline{\mathrm{u}}_{1}\left(\xi_{0}, \eta\right)+\rho_{1}\left(\xi_{0}, \eta\right) \\
& \mathrm{v}_{2}(\eta)=-\mathrm{a}_{0} \mathrm{u}_{1}\left(\xi_{0}, \eta\right)+\mathrm{u}_{1}\left(\xi_{0}, \eta\right) \\
& \mathrm{v}_{3}(\eta)=\rho_{0} \mathrm{a}_{0}^{2} \overline{\mathrm{u}}_{1}\left(\xi_{0}, \eta\right)+\mathrm{p}_{1}\left(\xi_{0}, \eta\right)
\end{aligned}
$$

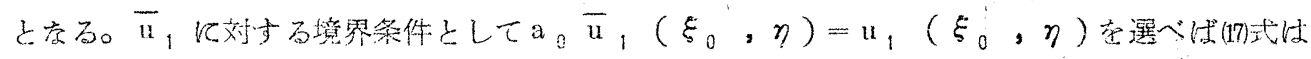

$$
\begin{aligned}
& \mathrm{v}_{1}(\eta)=\rho_{0}\left\{\bar{u}_{1}\left(\xi_{0}, \eta\right)+\bar{\rho}_{1}\left(\xi_{0}, \eta\right)\right\} \\
& v_{2}(\eta)=0 \\
& v_{3}(\eta)=\gamma p_{0}\left\{u_{1}\left(\xi_{0}, \eta\right)+\frac{1}{\gamma} p_{1}\left(\xi_{0}, \eta\right)\right.
\end{aligned}
$$

$(17)^{\prime}$

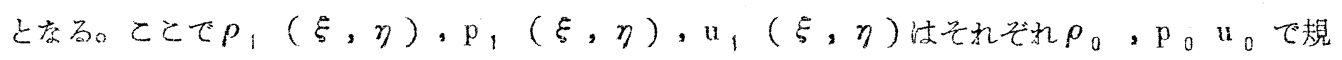

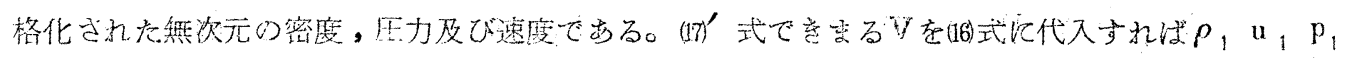
以未知量、だ壮で表方される。

$$
\left.\begin{array}{l}
\rho_{1}(\xi, \eta)=-\rho_{0} \bar{u}_{1}(\xi, \eta)+\rho_{0} \bar{u}_{1}(\xi, \eta)+\rho_{1}(\xi, \eta) \\
u_{1}(\xi, \eta)=a_{0} \bar{u}_{1}(\xi, \eta) \\
p_{1}(\xi, \eta)=-\rho_{0} a_{0}^{2} \bar{u}_{1}(\xi, \eta)+\rho_{0} a_{0}^{2} \bar{u}_{1}(\xi, \eta)+p_{1}(\xi, \eta) .
\end{array}\right\}
$$


Reductive Perturbation Method in Nonlinear Wave Propagation III. Formation and Developernent of Shock Waves in a Non-uniform Duct

$\bar{u}_{1}(\xi, \eta)$ 注次の上うK( ベクトル1左からかけると

$$
\bar{u}_{1 \eta}+\left(c_{1} \bar{u}_{1}+c_{2}\right) \bar{u}_{1 \xi}+c_{3}=0
$$

を得る。ここで係数は

$$
\begin{aligned}
c_{1} & =1 \cdot I\left(\nabla_{\mathrm{u}} \mathrm{A}\right)_{0} \cdot x / \mathrm{c}_{0}=\frac{r+1}{2} \mathrm{a}_{0} \\
c_{2} & =1 \cdot \mathrm{V}\left(\nabla_{\mathrm{u}} \mathrm{A}\right)_{0} \cdot \mathrm{I} / \mathrm{c}_{0} \\
& =\frac{1}{2} \mathrm{a}_{0}\left\{(1-r) \overline{\mathrm{u}}_{1}\left(\xi_{0}, \eta\right)-\bar{\rho}_{1}\left(\xi_{0}, \eta\right)+\bar{\rho}_{1}\left(\xi_{0}, \eta\right)\right\} \\
c_{3} & =\left(1 \mathrm{~V}_{\eta}+\frac{1}{\mathrm{~s}_{0}} \overline{\mathrm{s}}_{2} \xi 1 \cdot \mathrm{B}_{0}\right) / \mathrm{c}_{0} \\
& =-\frac{1}{2}\left\{\bar{u}_{1}\left(\xi_{0}, \eta\right)+\frac{1}{r} \bar{p}_{1 \eta}\left(\xi_{0}, \eta\right)\right\}-\frac{1}{2} \mathrm{a}_{0} \frac{\mathrm{s}_{2} \xi}{\mathrm{s}_{0}} \\
c_{0} & \equiv 1 \Gamma_{0} \mathrm{x}=\frac{2}{\gamma-1} \rho_{0} \mathrm{a}_{0}^{2}
\end{aligned}
$$

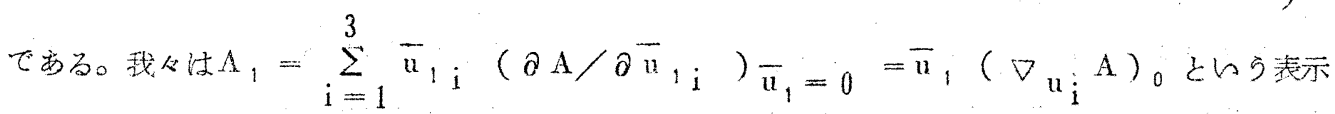

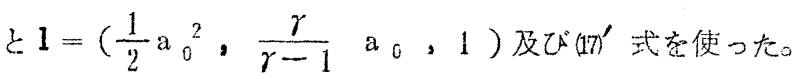

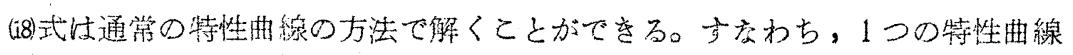

$$
\frac{d \xi}{d \eta}=c_{1} \bar{u}_{1}+c_{2}
$$

上で

$$
\overline{\mathrm{u}}_{i}+\int \mathrm{c}_{3} \mathrm{~d} \eta=\mathrm{c}=\mathrm{const} .
$$

が成立する。

(21) 式加速度 $\overline{\mathrm{u}}$ 、壮

$$
\overline{\mathrm{u}}_{1}=\mathrm{c}-\int \mathrm{c}_{3} \mathrm{~d} \eta
$$


で与えられるから(19)式の $c_{3}$ の各項が、、にどのよう度効果を与えるか見よう。立ず流体のマッハ 数Mr土 $\mathrm{u}_{0}=\mathrm{a}_{0} \mathrm{~K}$ 注意して

$$
\mathrm{M}=\frac{\mathrm{u}}{\mathrm{a}}=1+\epsilon\left(\overline{\mathrm{u}}_{1}-\overline{\mathrm{a}}_{1}\right)+\cdots
$$

となる。以下では $M_{1} \equiv \bar{u}_{1}-\bar{a}_{1}$ をマッ八数と烀ぶ。これは(16)’ 式から

$$
M_{1}(\xi, \eta)=\frac{\gamma+1}{2}\left\{\bar{u}_{1}(\xi, \eta)-\bar{u}_{1}\left(\xi_{0}, \eta\right)\right\}+M_{1}\left(\xi_{0}, \eta\right)
$$

と書くことがで音る。このマッハ数孛使うと(19)(20)式から

$$
\frac{d \xi}{d \eta}=a_{0} M_{1}(\xi, \eta)
$$

$(20)^{\prime}$

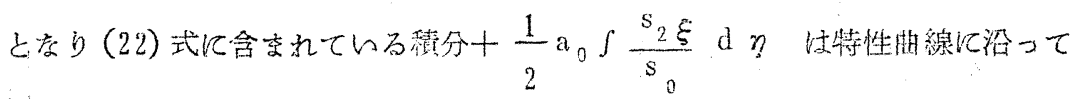

$$
+\frac{1}{2} \frac{1}{s_{0}} \int \frac{1}{M_{1}} s_{2} \xi d \xi
$$

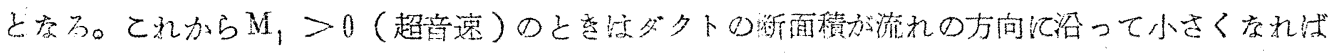

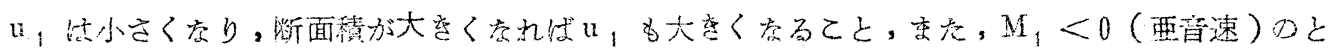
をは事情が逆化志るととがわかる。

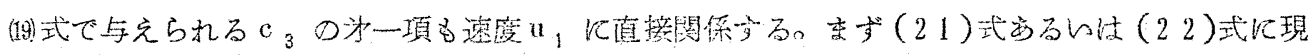

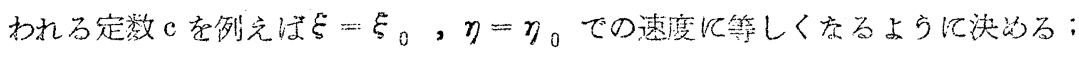

$$
c=\bar{u}_{1}\left(\xi_{0}, \eta_{0}\right) \text {. }
$$

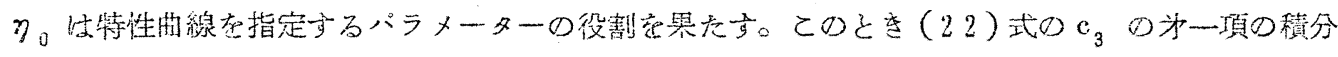
袁含む湏壮

$$
+\frac{1}{2}\left[\bar{u}_{1}\left(\xi_{0} \eta\right)+\frac{1}{\gamma} \bar{p}_{1}\left(\xi_{0} \eta\right)\right] \begin{aligned}
& \eta \\
& \eta=\eta \\
& \eta=\eta_{0}
\end{aligned}
$$

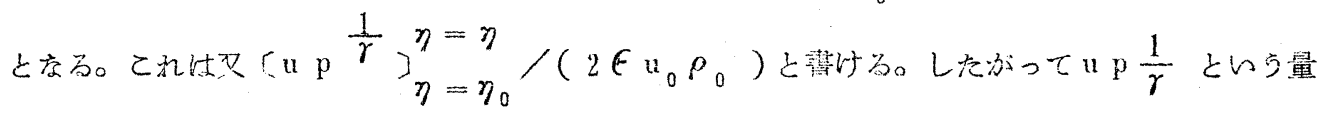

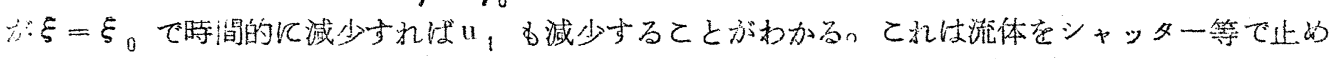
ようとするとを起る現笔を表わす。 
Reductive Perturbation Method in Nonlinear Wave Propagation III. Formation and Developement of Shock Waves in a Non-uniform Duct

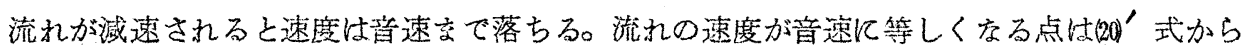

$$
\frac{d \xi}{d \eta}=0
$$

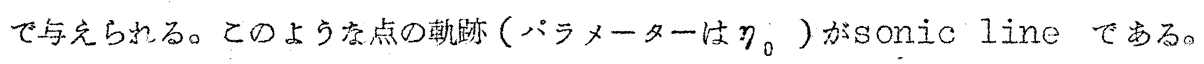

$\S 3$ 円錐形のconvergence を持ったダクトの場合

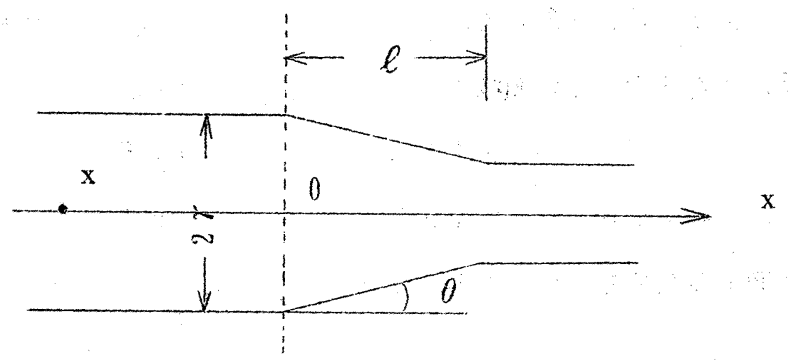

图 2 convergence の无デッ

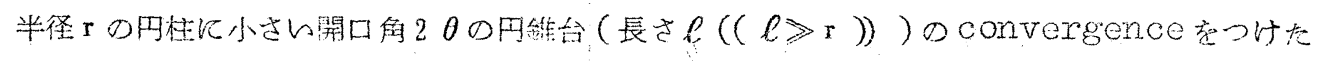

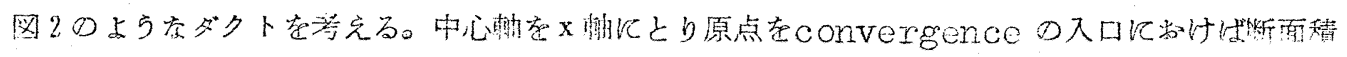
心は次のようになる。

$$
s= \begin{cases}s_{0} & (x \leqq 0) \\ s_{0}\left(1-\theta \frac{x}{r}\right)^{2} & (0 \leqq x \leqq \ell) \\ s_{0}\left(1-\theta \frac{\ell}{r}\right)^{2} & (x \geq \ell)\end{cases}
$$

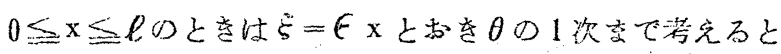

$$
\begin{aligned}
\mathrm{s} & =\mathrm{s}_{0}-2 \pi \mathrm{r} \dot{\theta} \mathrm{x} \\
& =\mathrm{s}_{0}+\xi^{2} \mathrm{~s}_{2}
\end{aligned}
$$

か心

$$
\epsilon=\theta^{\frac{1}{3}}
$$


おょび

$$
\mathrm{s}_{2}=-2 \pi \mathrm{r} \xi
$$

を得る。（22）(24）(25)および（27）式からとの領域では

$$
\begin{aligned}
\overline{\mathrm{u}}_{1}= & \overline{\mathrm{u}}_{1}\left(\xi_{0}, \eta_{0}\right)+\frac{1}{2}\left[\overline{\mathrm{u}}_{1}\left(\xi_{0}, \eta\right)+\frac{1}{r} \overline{\mathrm{p}}_{1}\left(\xi_{0}, \eta\right)\right] \begin{array}{l}
\eta=\eta \\
\eta=\eta_{0}
\end{array} \\
& -\frac{\mathrm{a}_{0}}{\mathrm{r}}\left(\eta-\eta_{0}\right)
\end{aligned}
$$

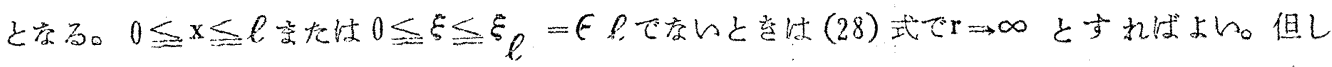

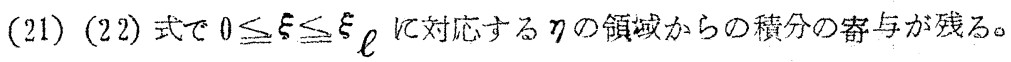

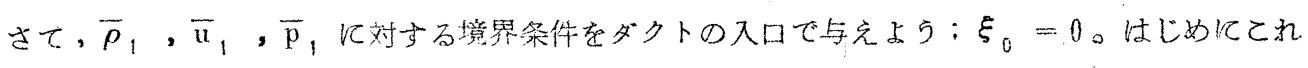

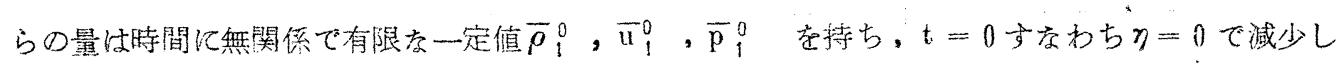

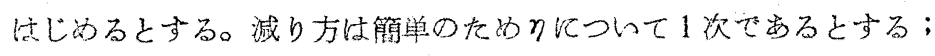

$$
\left.\begin{array}{r}
\bar{\rho}_{1}(0, \eta)=\bar{\rho}_{1}^{0} \quad-\mathrm{m}_{\rho} \eta \\
\bar{u}_{1}(0, \eta)=\overline{\mathrm{u}}_{1}^{0} \quad-\mathrm{m}_{\mathrm{u}} \eta \\
\overline{\mathrm{p}}_{1}(0, \eta)=\overline{\mathrm{p}}_{1}^{0}-\mathrm{m}_{\mathrm{p}} \eta
\end{array}\right\}
$$

と宗。

$$
\mathrm{m}^{0} \equiv \overline{\mathrm{u}}_{1}^{0}-\frac{1}{2}\left(\overline{\mathrm{p}}_{1}^{0}-\bar{\rho}_{1}^{0}\right)
$$

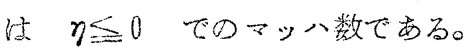

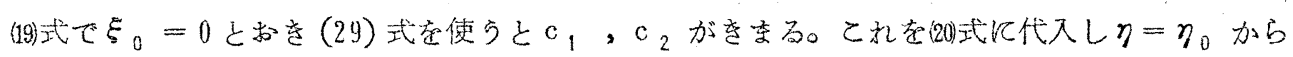

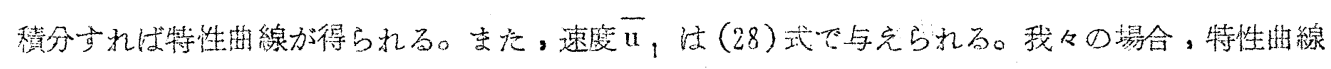

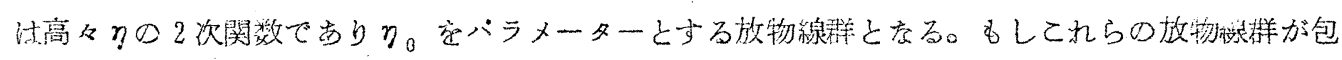

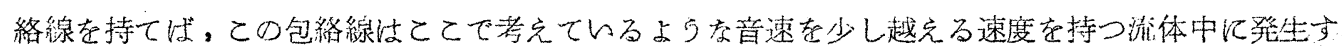

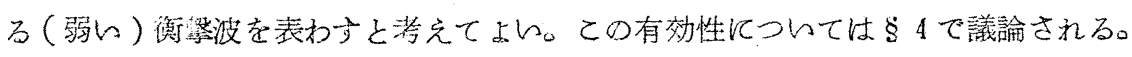

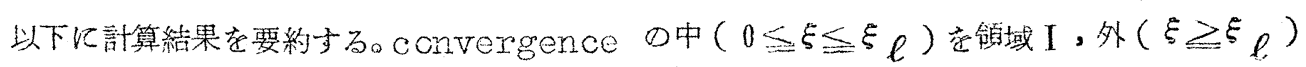


Reductive Perturbation Method in Nonlinear Wave Propagation III. Formation and Developement of Shock Waves in a Non-uniform Duct 意領域にとしょう。後でわかるように convergenceが $0^{0^{2}} \mathrm{r} /(\gamma+1)$ より長いと conver一 gence つ中绽在的不連続面がでさるしたがって，条件

$$
\xi_{\ell} \geq \frac{\mathrm{m}^{0^{2}}}{r+1} \mathrm{r}
$$

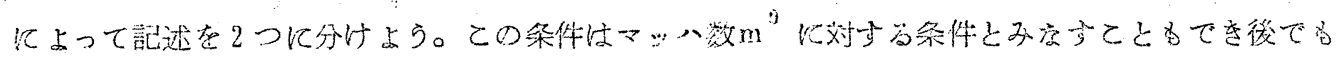
ら一度ふれる。

a) $\xi_{\ell} \geq \frac{m^{0^{2}}}{r+1} \mathrm{r}$. のとき。

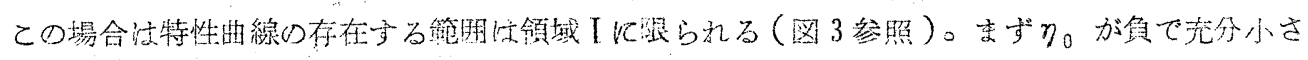

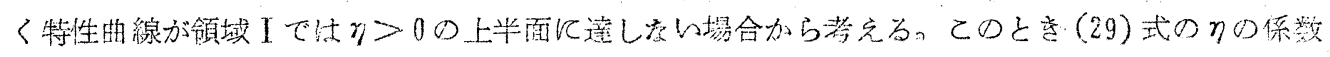

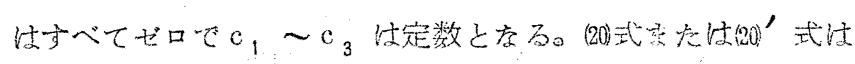

$$
\frac{\mathrm{d} \xi}{\mathrm{d} \eta}=a_{0} m^{0},-\frac{r+1}{2} \frac{\mathrm{a}_{0}^{2}}{\mathrm{r}}\left(\eta-\eta_{0}\right)
$$

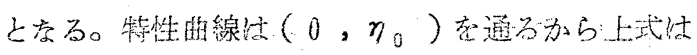

$$
\xi=a_{0}\left(\eta-\eta_{0}\right)\left\{m^{0}-\frac{\gamma+1}{4 r} a_{0}\left(\eta-\eta_{0}\right)\right\}
$$

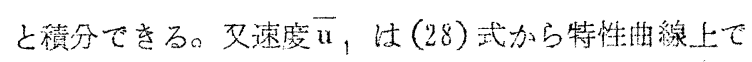

$$
\bar{u}_{1}=\bar{u}_{1}^{0} \quad-\frac{\mathrm{a}_{0}}{\mathrm{r}}\left(\eta-\eta_{0}\right)
$$

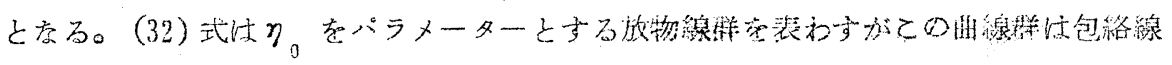

$$
\xi=\frac{\mathrm{m}^{0^{2}}}{r+1} \quad \mathrm{r}=\mathrm{cons} \mathrm{t}
$$

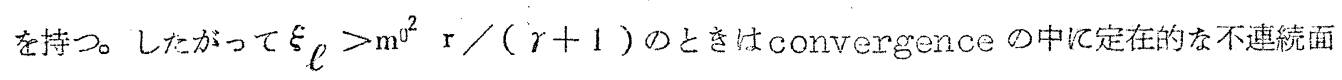

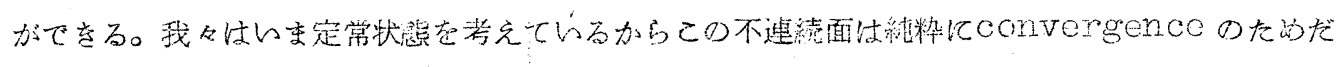

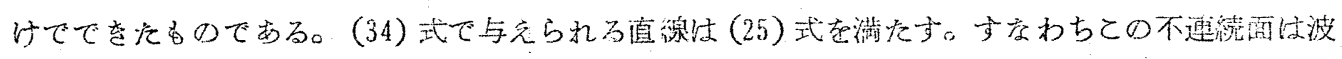
が音速になって䚻ってくる面を表している。

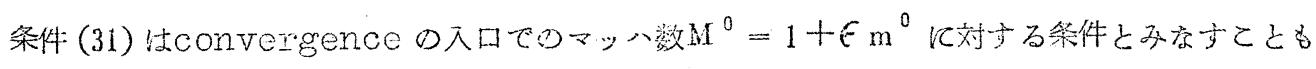




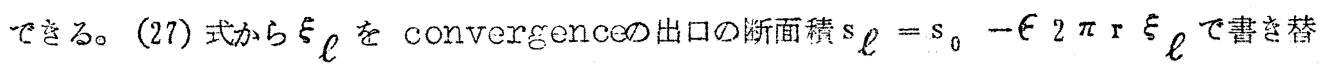
えると（31）式は

$$
\mathrm{M}^{0} \leqq 1+\left\{\frac{\gamma+1}{2}\left(1-\frac{\mathrm{s} \ell}{\mathrm{s}_{0}}\right)\right\}^{\frac{1}{2}}
$$

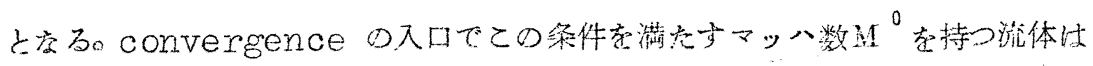

$$
\frac{S}{S_{0}}=1-\frac{2}{r+1}\left(M^{0}-1\right)^{2}
$$

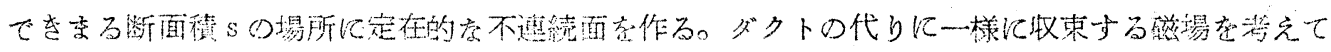

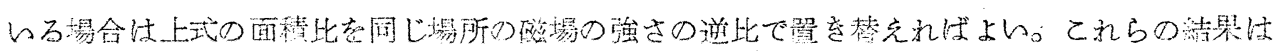
Hamada et. al. 54) Leloup and Taussig 等の綘寨之もの二次のオーがー妾で 一秌与方。

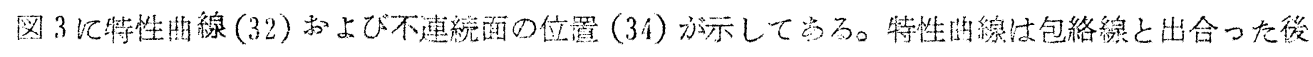

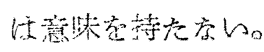

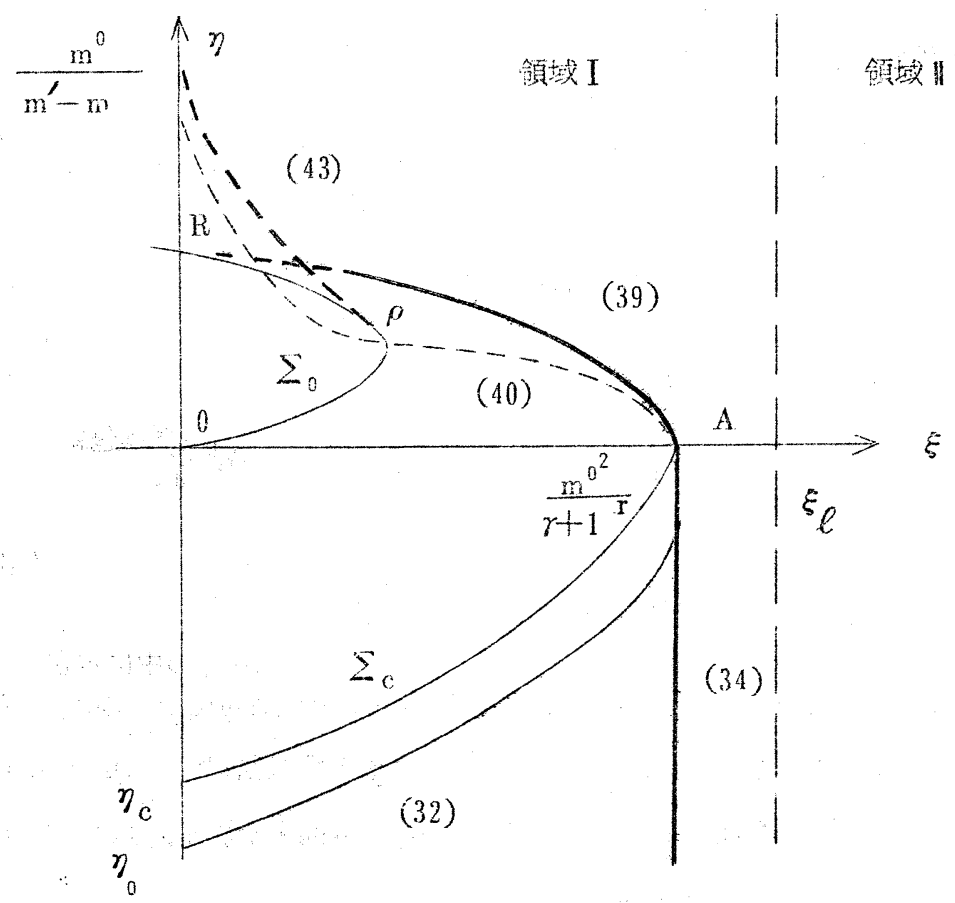

図3衡慗波の唄動(1) 
Reductive Perturbation Method in Nonlinear Wave Propagation III. Formation and Developenent of Shock Waves in a Non-uniform Duct

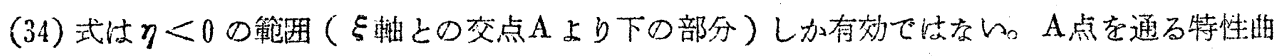
線恃

$$
\eta_{0}=\eta_{c} \equiv \frac{-2}{r+1} \frac{r}{a_{0}}
$$

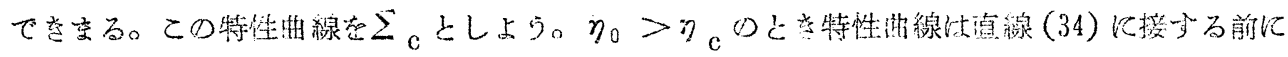
$\eta>0$ 部分に入る。

$$
\xi_{\alpha}=-a_{0} \eta_{0}\left(m^{0}+\frac{\gamma+1}{4 r} a_{0} \eta_{0}\right)
$$

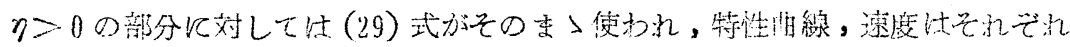

$$
\begin{aligned}
& \xi-\xi_{\alpha}=a_{0} \eta\left\{m^{0}+\frac{r+1}{2 r} a_{0} \eta_{0}+\left(m-\frac{r+1}{4 r} a_{0}\right) \eta\right\} \\
& \bar{u}_{1}=\bar{u}_{1}-\frac{1}{2}\left(m_{u}+\frac{1}{r} m_{p}\right) \eta-\frac{a_{0}}{r}\left(\eta-\eta_{0}\right)
\end{aligned}
$$

と奆っこてで仗

$$
m \equiv \frac{1}{4}\left(\frac{\gamma-3}{2} m_{u}+\frac{\gamma-1}{2 \gamma} m_{p}-m_{\rho}\right)
$$

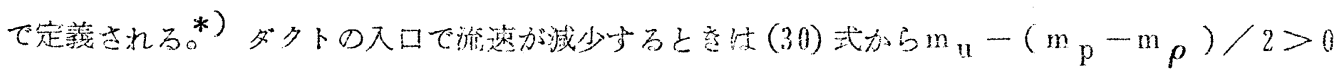

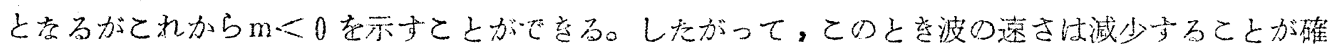

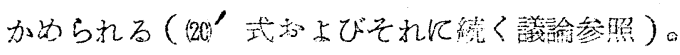

（36）式は（35）式老倠って

$$
\xi=a_{0}\left\{m^{0}\left(\eta-\eta_{0}\right)+\frac{r+1}{4 \mathrm{r}} \mathrm{a}_{0} \eta_{0}\left(2 \eta-\eta_{0}\right)+\left(m-\frac{r+1}{4 \mathrm{r}} \mathrm{a}_{0}\right) \eta^{2}\right\}
$$

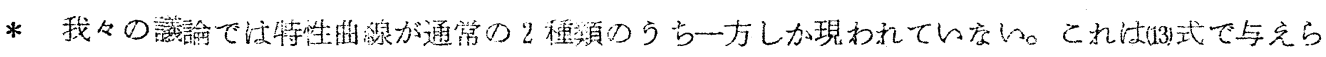
れる stretchingの結框起ったととで，う一方の特性曲線はd $\xi / \mathrm{d} \eta 2_{2} \mathrm{a}_{0} / \varepsilon$ と

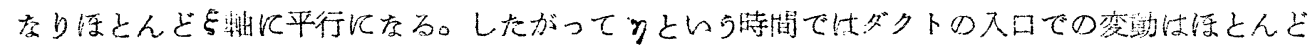

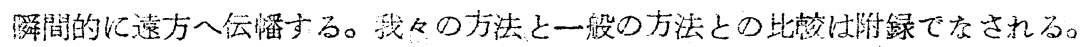




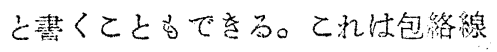

$$
\xi=\frac{\mathrm{m}^{0^{2}}}{r+1} \mathrm{r}+\mathrm{a}_{0} \mathrm{~m} \eta^{2}
$$

在持つ。ての包絡線な点Aで（34）式で与えられる直線と疗的らかに接している。 壮 (26) 式加ら (36) '式の頂点の軦跡として決的られ

$$
\xi=\frac{\mathrm{m}^{0^{2}}}{r+1} \mathrm{r}-\frac{4 \mathrm{r}}{\gamma+1} \mathrm{~m}\left(\mathrm{~m}-\frac{\gamma+1}{4 \mathrm{r}} \mathrm{a}_{0}\right) \eta^{2}
$$

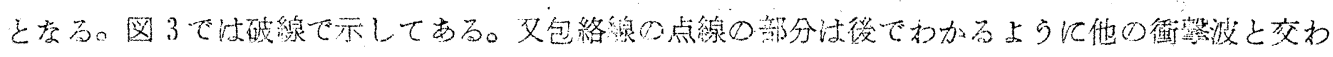

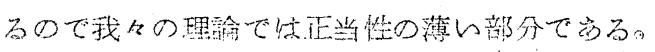

(35) 〜 (37) 式，したがって (36) $)^{\prime}(39)$ 式は $\eta_{0}=0$ 宫で有効である。 $\eta_{0}=0$ である特性曲

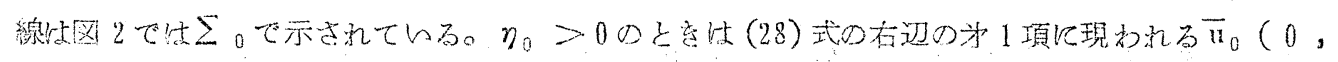

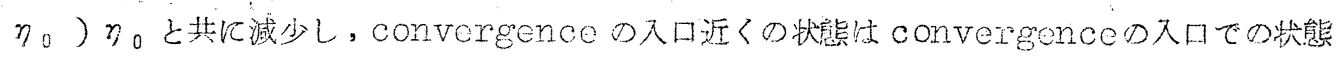

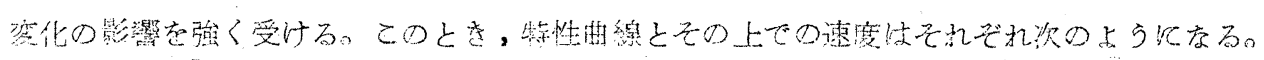

$$
\begin{aligned}
& \xi=a_{0}\left\{\eta-\eta_{0}\right)\left\{m^{0}-\left(m^{\prime}-\frac{\gamma+1}{4 r} a_{0}\right) \eta_{0}+\left(m-\frac{r+1}{4 r} a_{0}\right) \eta\right\} \\
& \bar{u}_{1}=\bar{u} \eta-m_{u} \eta_{0}-\frac{1}{2}\left(m_{u}+\frac{1}{r} m_{p}\right)\left(\eta-\eta_{0}\right)-\frac{a_{0}}{r}\left(\eta-\eta_{0}\right)
\end{aligned}
$$

ここで

$$
m^{\prime}=m+\left\{m_{u}--\left(m_{p}-m_{\rho}\right)\right\}
$$

だる。（41）式は

$$
\xi=a_{0} M_{1}\left(0, \eta_{0}\right)\left(\eta-\eta_{0}\right)+a_{0}\left(m-\frac{r+1}{4 r} a_{0}\right)\left(\eta-\eta_{0}\right)^{2}
$$

と萻くこともでさる。（41）式壮包絡線

$$
\xi=-\frac{a_{0}}{4} \frac{\left(m^{\prime}-m\right)^{2}}{m^{\prime}-\frac{r+1}{4 r} a_{0}}\left(\eta-\frac{m^{0}}{m^{\prime}-m}\right)^{2}
$$

を持つ。右辺の最後の項に含をれる $\frac{\mathrm{m}^{0}}{\mathrm{~m}^{\prime}-\mathrm{m}}$ は $\mathrm{m}^{\prime}$ の定義(43)式と(30)式からわかるようにダ 
Reductive Perturbation Methoa in Nonlinear Wave Propagation III. Formation and Developement of Shock Waves in a Non-miform Duct

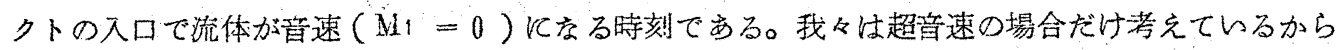

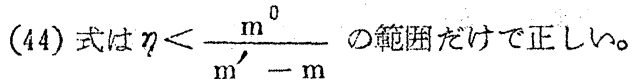

$$
\text { さて, } m^{\prime}-\frac{\gamma+1}{4 \mathrm{r}} \mathrm{a}_{0}<0 \text { 度ら（44）式は } \xi>00 \text { 部分（領域 I ）に含去れ }
$$

$\mathrm{m}^{\prime}-\frac{r+1}{4 \mathrm{r}} \mathrm{a}_{0}>0$ 症領域 IKは存在し索的ししがって

$$
\mathrm{m}^{\prime}-\frac{r+1}{4 \mathrm{r}} \mathrm{a}_{0}<0
$$

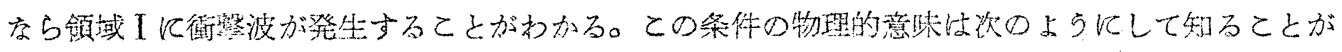

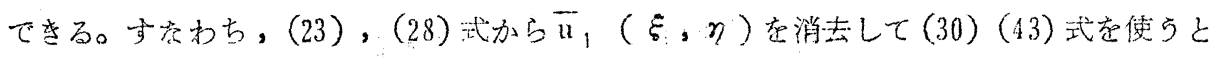

$$
M_{1}(\xi, \eta)-M_{1}\left(\xi_{0}, \eta\right)+\frac{\gamma+1}{4 \mathrm{r}} \mathrm{a}_{0}\left(\eta-\eta_{0}\right)=\left(\mathrm{m}^{\prime}-\frac{\gamma+1}{4 \mathrm{r}} \mathrm{a}_{0}\right)\left(\eta-\eta_{0}\right)
$$

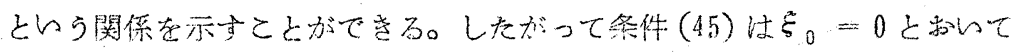

$$
M_{1}(\xi, \eta)<M_{1}(0, \eta)-\frac{r+1}{4 r} a_{0}\left(\eta-\eta_{0}\right)
$$

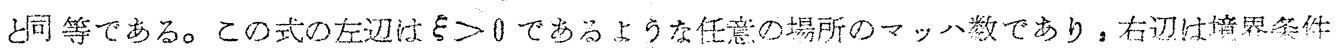
として状態が上充られた場所 $\xi=0$ でマッ八数から波がconvergence か中伝わっている時

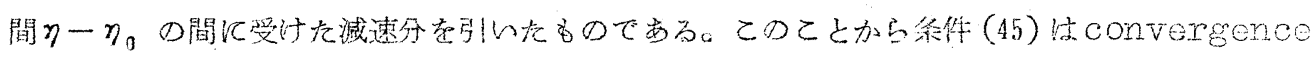

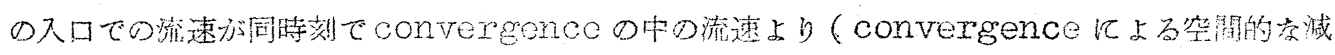

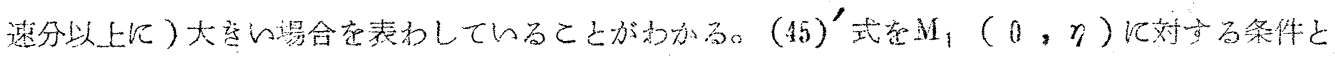

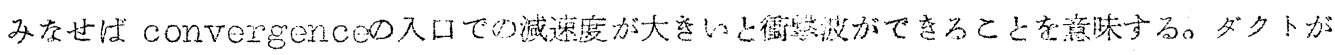

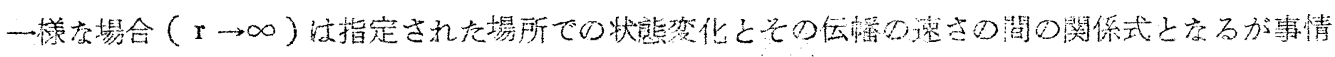
は変ら灰的。

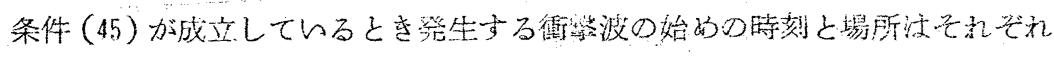

$$
\left.\begin{array}{l}
\eta_{p}=\frac{m^{0}}{1 m+m^{\prime}-\frac{r+1}{2 r} a_{0} 1} \\
\xi_{p}=a_{0}\left(m^{\prime}-\frac{r+1}{4 r} a_{0}\right) \frac{m-m^{\prime}}{m^{0}} \eta_{p}^{2}
\end{array}\right\}
$$




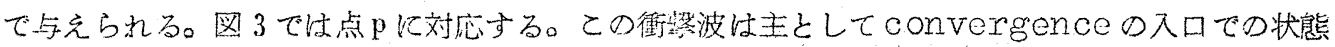

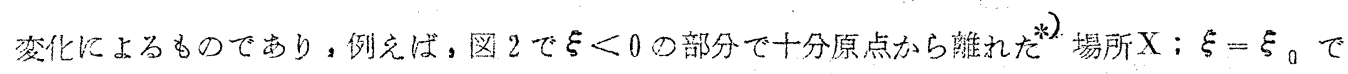

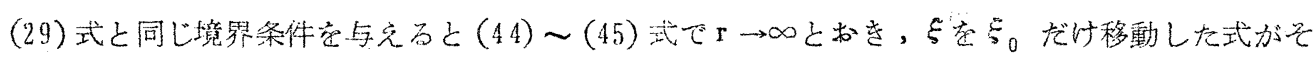

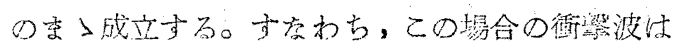

$$
\begin{aligned}
& \eta_{p}^{\prime}=\frac{m^{0}}{1 m+m^{\prime} 1} \\
& \xi_{p}^{\prime}=\xi_{0}+a_{0} \frac{m^{\prime}\left(m-m^{\prime}\right)}{\left(m+m^{\prime}\right)^{2}} m^{0}
\end{aligned}
$$

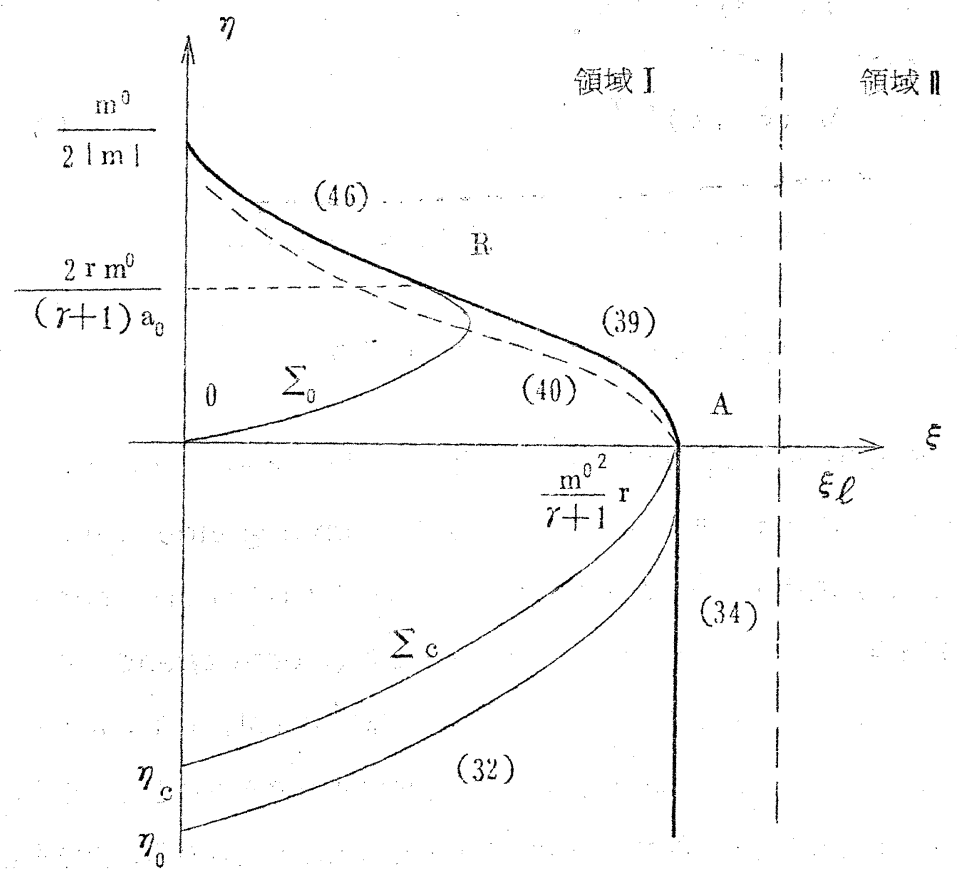

図 4 衡浖波の運動(2)

* 実際壮 $\eta=0 て ゙ \xi=\xi 。$ を出る特性曲線が原点 0 に㵆しない条件

$$
\xi_{0}<\frac{\mathrm{a}_{0}}{4} \frac{\mathrm{m}^{0^{2}}}{\mathrm{~m}}
$$

が成立すればよい。 
Reductive Perturbation Method in Nonlinear Wave Propagation III. Formation and Developement of Shock Waves in a Non-uniform Duct

で発生する。またconvergenceの中の衙勘波恃図4のようになるての図で

$\eta \geq \frac{2 \mathrm{r}}{r+1} \frac{\mathrm{m}^{0}}{\mathrm{a}_{0}}$ の部分の包絡線は

$$
\xi=a_{0} \frac{m^{2}}{m+\frac{r+1}{4 r} a_{0}}\left(\eta+\frac{m^{0}}{2 m}\right)^{2}
$$

で表わされる。

b) $\xi_{\ell} \leqq \frac{m^{0^{2}}}{r+1} \mathrm{r}$ oと ( $_{0}^{*}$

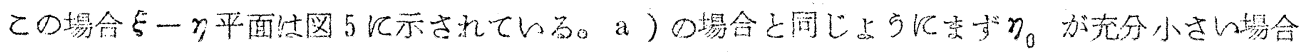

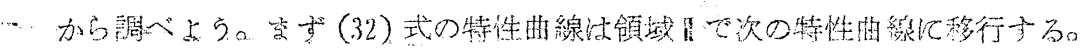

$$
\xi-\xi_{\ell}=a_{0}\left\{m^{0}-\frac{\gamma+1}{2 r} a_{0}\left(\eta_{\alpha}-\eta_{0}\right)\right\}\left(\eta-\eta_{\alpha}\right)
$$

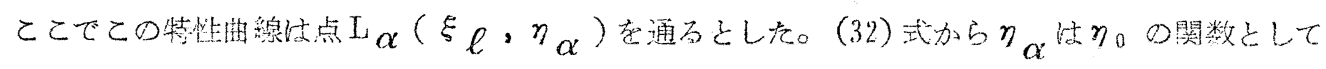

$$
\xi_{\ell}=\mathrm{a}_{0}\left(\eta_{\alpha}-\eta_{0}\right)\left\{\mathrm{m}^{0}-\frac{\gamma+1}{4 \mathrm{r}} \mathrm{a}_{0}\left(\eta_{\alpha}-\eta_{0}\right)\right\}
$$

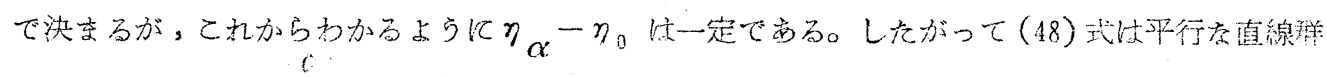

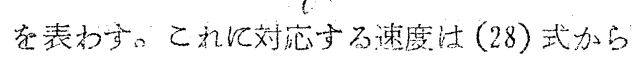

$$
\bar{u}_{1}=\bar{u}_{1}^{0}-\frac{a_{0}}{\mathrm{r}}\left(\eta_{\alpha}-\eta_{0}\right)
$$

と疗る。

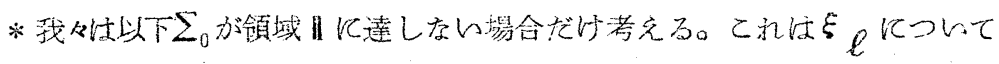

$$
\xi \ell^{>-\frac{a_{0}}{4}} \frac{m^{2}}{m-\frac{r+1}{4 r} a_{0}}
$$

が成立する場合である(眓 5 参照)。 


\section{浅 野}

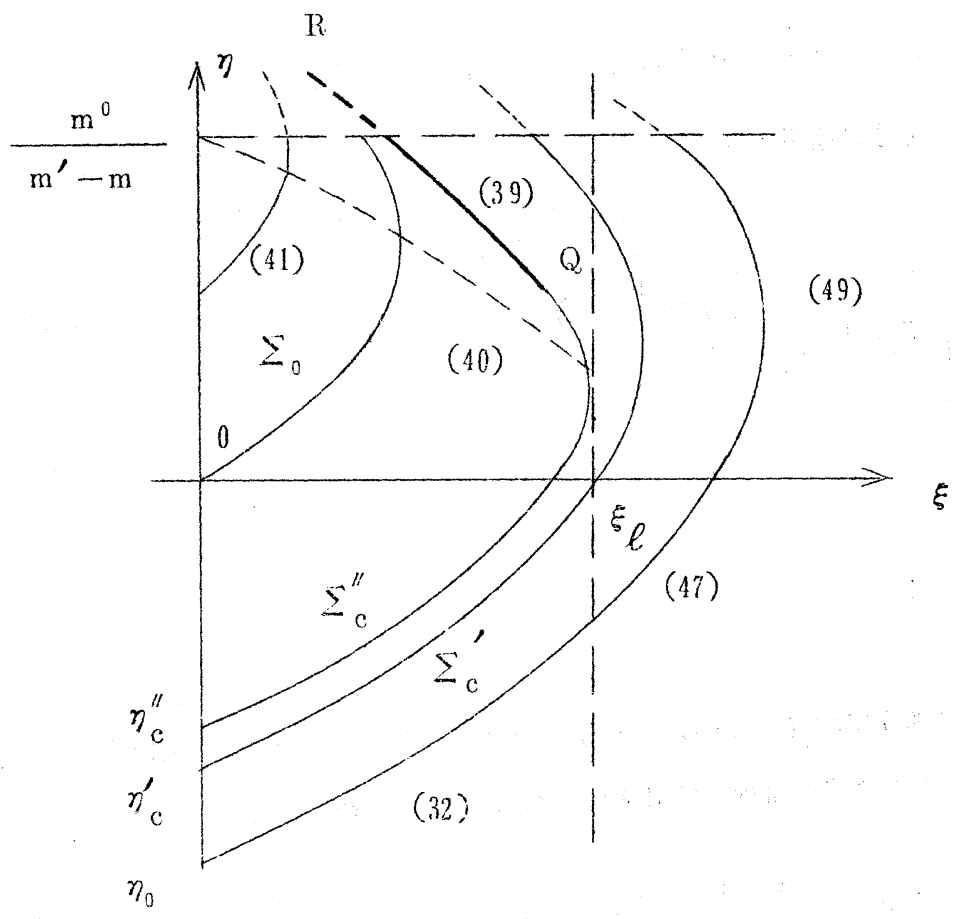

図 5 衡涂波の渾動(引)

（48）式の特性曲線で表わされる波仕クニnのとを

$$
\xi=\xi_{\beta}=\xi_{\ell}-a_{0}\left\{m^{0}-\frac{\gamma+1}{2 r} a_{0}\left(\eta_{\alpha}-\eta_{0}\right)\right\} \eta_{\alpha}
$$

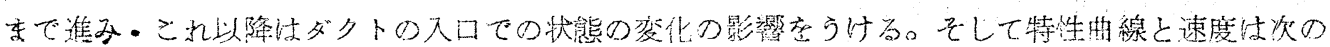
上うになる。

$$
\xi-\xi_{\beta}=a_{0}\left\{m^{0}-\frac{\gamma+1}{2 r} a_{0}\left(\eta_{\alpha}-\eta_{0}\right)+m \eta\right\} \eta
$$

又沬 $\xi \beta$ 然消去して

$$
\xi-\xi e^{-a_{0}}\left\{m^{0}-\frac{r+1}{2 r} a_{0}\left(\eta \alpha-\eta_{0}\right)\right\}\left(\eta-\eta_{\alpha}\right)+a_{0} m \eta^{2}(50)^{\prime}
$$

故び 
Reductive Perturbation Method in Nonlinear Wave Propagation III. Formation and Developement of Shock Waves in a Non-uniform Duct

$$
\bar{u}_{1}=\bar{u}_{1}-\frac{a_{0}}{r}\left(\eta_{\alpha}-\eta_{0}\right)-\frac{1}{2}\left(m_{u}+\frac{1}{r} m_{p}\right) \eta .
$$

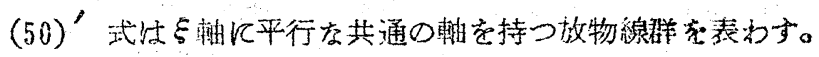

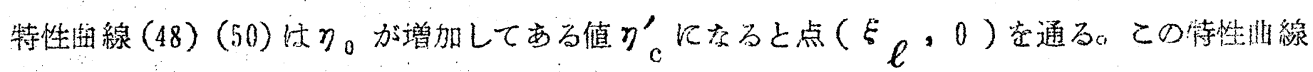

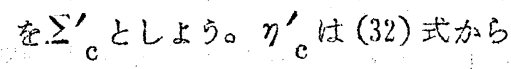

$$
\xi_{\ell}=-\mathrm{a}_{0} \eta_{\mathrm{c}}^{\prime}\left(\mathrm{m}^{0}+\frac{r+1}{4 \mathrm{r}} \mathrm{a}_{0} \eta_{\mathrm{c}}^{\prime}\right)
$$

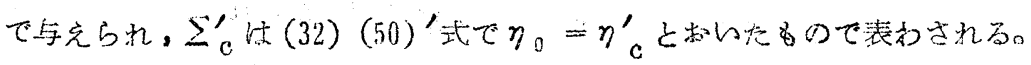

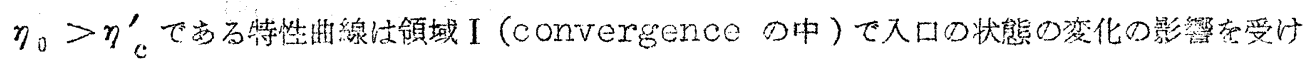

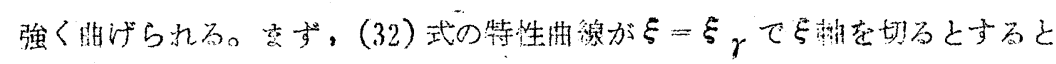

$$
\xi_{\gamma}=-a_{0} \eta_{0}\left(m^{0}+\frac{r+1}{4 r} a_{0} \eta_{0}\right)
$$

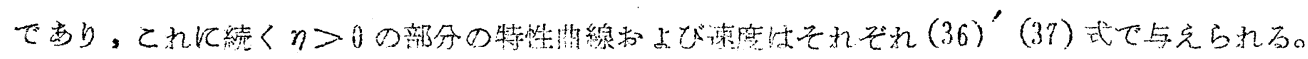

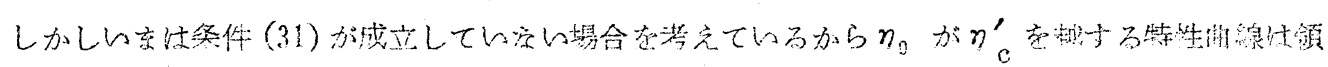

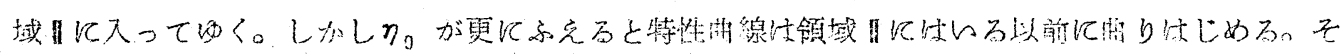

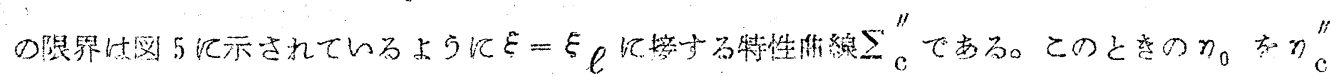

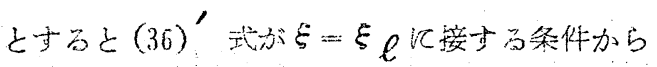

$$
\eta_{\mathrm{c}}^{\prime \prime}=\frac{\frac{1}{r+1} \frac{-r}{\mathrm{~m}}\left\{2 \mathrm{~mm} \mathrm{~m}^{0}+\sqrt{4 \mathrm{~m}^{2} \mathrm{~m}^{0^{2}}-\frac{\mathrm{r}+1}{\mathrm{r}} \mathrm{a}_{0} \mathrm{~m}\left(\mathrm{~m}^{0^{2}}+4 \xi \ell\right)}\right.}{\quad \frac{\left.\left(\mathrm{m}-\frac{r+1}{4 \mathrm{r}} \mathrm{a}_{0}\right)\right\}}{}}
$$

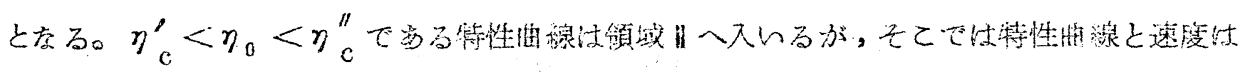

$$
\begin{aligned}
& \xi-\xi_{\ell}=a_{0}\left(\eta-\eta_{\beta}\right)\left\{m^{0}-\frac{\gamma+1}{2 r} a_{0}\left(\eta_{\beta}-\eta_{i}\right)+m \eta_{\beta}+m \eta\right\} \\
& \vec{u}_{1}=\vec{u}_{1}^{0}-\frac{a_{0}}{r}\left(\eta_{\beta}-\eta_{0}\right)-\frac{1}{2}\left(m_{u}+\frac{1}{r} m_{p}\right) \eta
\end{aligned}
$$




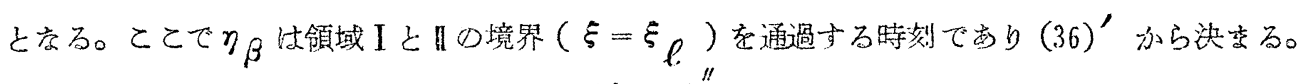

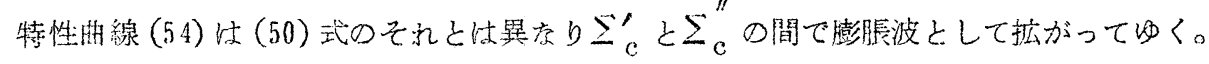

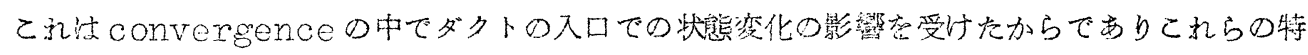

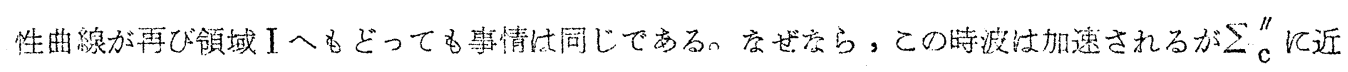

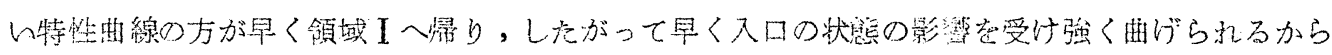
である。

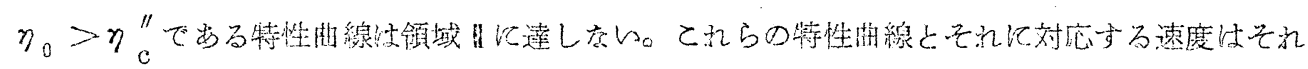

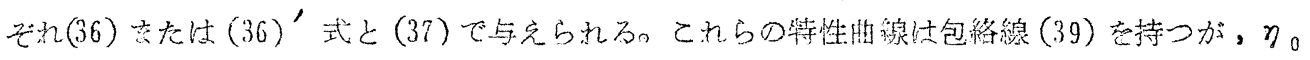

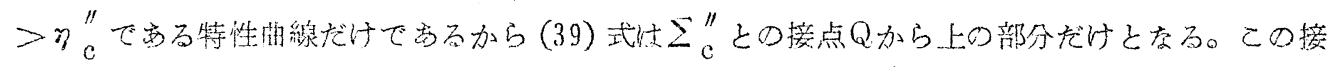
点の空標忛

$$
\begin{aligned}
& \xi_{\mathrm{Q}}=\frac{\mathrm{r}}{r+1} \frac{\mathrm{m}^{0}}{\mathrm{a}_{0}}+\mathrm{m}\left(\eta_{\mathrm{c}}^{\prime \prime}+\frac{2 \mathrm{rm} \mathrm{m}^{0}}{r+1} \frac{1}{\mathrm{a}_{0}}\right)^{2} \\
& \eta_{\mathrm{Q}}=\eta_{\mathrm{c}}^{\prime \prime}+\frac{2 \mathrm{rm} \mathrm{m}^{0}}{r+1} \frac{1}{\mathrm{a}_{0}}
\end{aligned}
$$

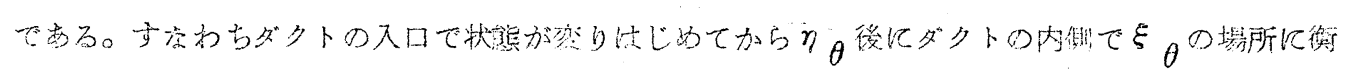

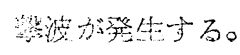

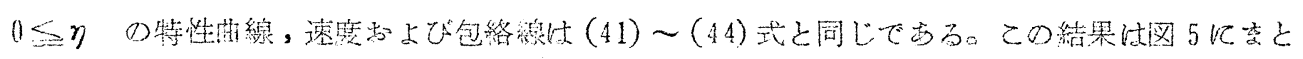

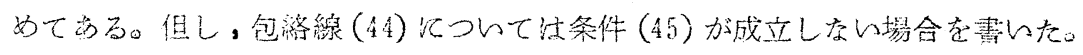

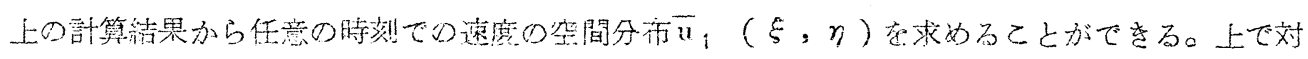

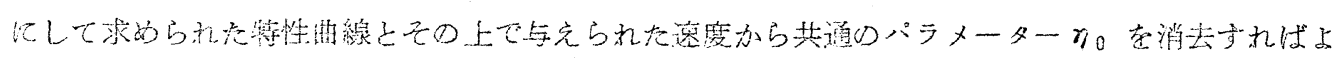

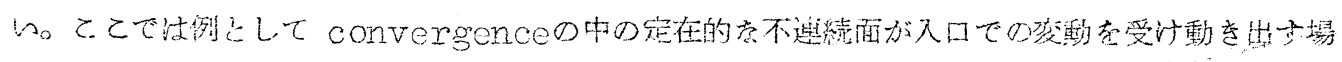

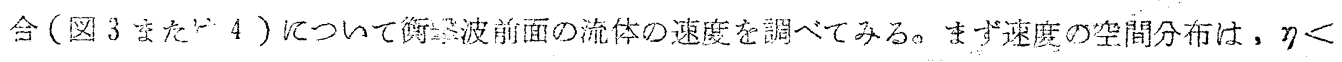
0のとを(32) (33) 式から

$$
\xi=r m^{0}\left(\bar{u}_{1}^{0}-\bar{u}_{1}\right)-\frac{r+1}{4} r\left(\bar{u}_{1}^{0}-\bar{u}_{1}\right)^{2}
$$

で李り, ク>0のと走は (36)' (37) 式から 
Reductive Perturbation Method in Nonlinear Wave Propagaiion III. Formation and Developement of Shock Waves in a Non-uniform Duct

$$
\begin{aligned}
& \xi-\left(\frac{\mathrm{m}^{0^{2}}}{r+1} \mathrm{r}+\mathrm{am} \eta^{2}\right)=-\frac{r+1}{4} \mathrm{r}\left\{\overline{\mathrm{u}}_{1}-\overline{\mathrm{u}}_{1}^{0}+\frac{1}{2}\left(\mathrm{~m}_{\mathrm{u}}+\frac{1}{r} \mathrm{~m}_{\mathrm{p}}\right) \eta\right. \\
& \left.+\frac{2}{r+1} \mathrm{~m}^{0}\right\}^{2}
\end{aligned}
$$

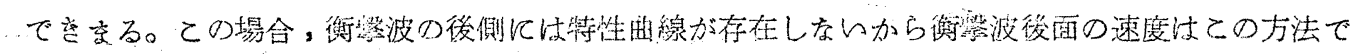

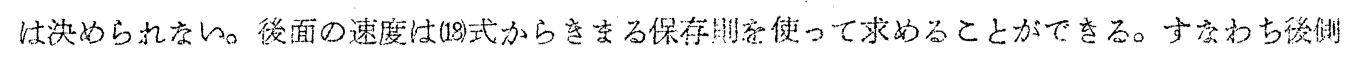

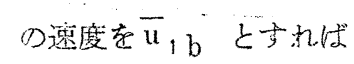

$$
\bar{u}_{1 b}=-\bar{u}_{1}+\frac{z}{c_{1}}\left(\lambda-c_{2}\right)
$$

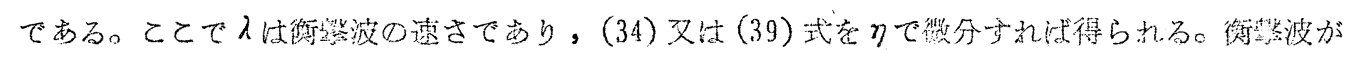

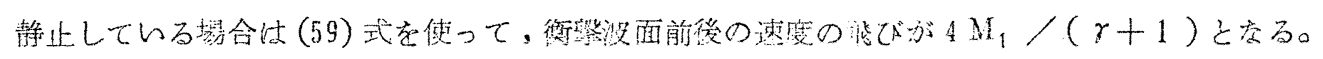
$M_{1}$ 愔前面のマッ八数でちる。

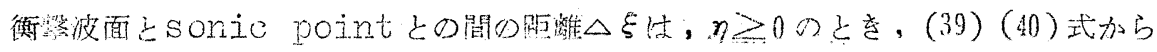

$$
\Delta \xi=\xi \text { shock }-\xi_{\text {sonic }}=\frac{4 \mathrm{r}}{\gamma+1} \mathrm{~m}^{2} \eta^{2} \text {. }
$$

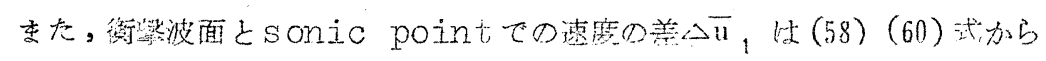

$$
\Delta \bar{u}_{1}=\bar{u}_{1 \text { shock }}-\bar{a}_{1}=-\frac{4}{\gamma+1} m \eta
$$

となり，平均の䢞庭勾配注

$$
\frac{\Delta \bar{u}_{1}}{\Delta \bar{\xi}}=-\frac{1}{m r \eta}
$$

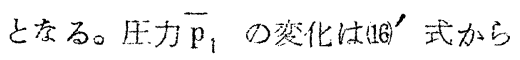

$$
\frac{\Delta \bar{p}_{1}}{\Delta \xi}=-r \frac{\Delta \bar{u}_{1}}{\Delta \xi}=\frac{r}{m r \eta}
$$

である。 


\section{$\$ 4$ 宗とめ}

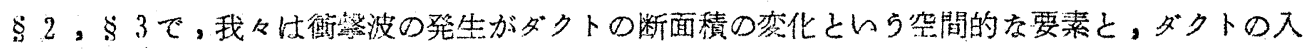

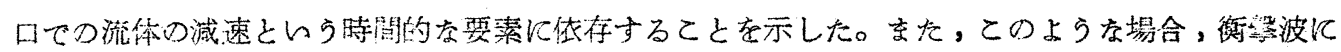
は二種類あり，一つ注入口での状热が定常的裳とさ convergenceの中で静止しており，入口の

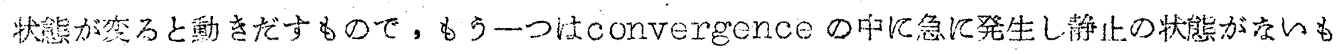

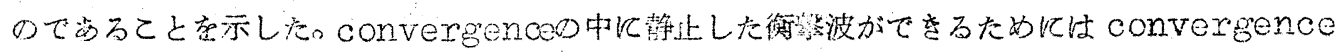
○琵さとが条仵

$$
\ell>\frac{m^{0^{2}}}{r+1} \frac{r}{\theta^{1} / 3}
$$

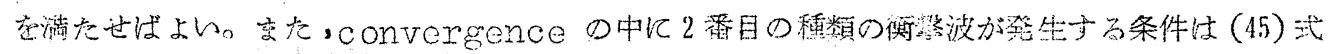

$$
m^{\prime}-\frac{r+1}{4 r} a_{0}<0
$$

である。

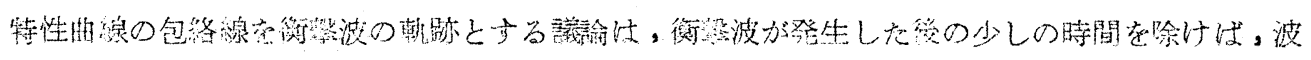

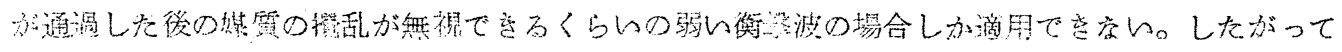

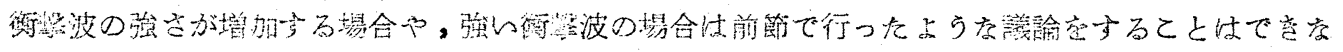
no

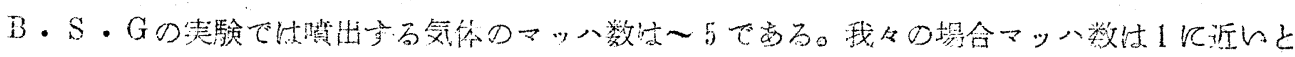

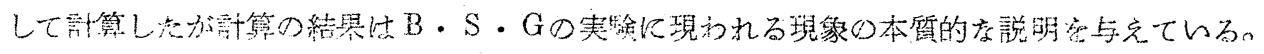

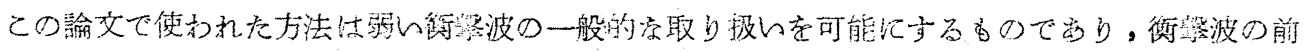

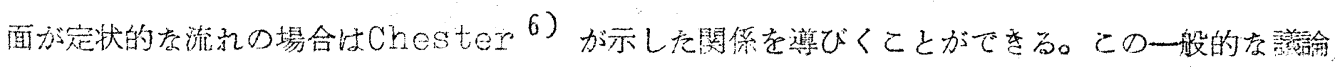
は現在進行中である。

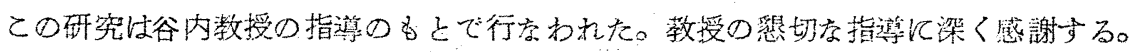


Reductive Perturbation Method in Nonlinear Wave Propagation III. Formation and Developement of Shock Waves in a Non-uniform Duct

\section{参考文献}

1. WHITHAM, G.B. $1968 \mathrm{~J}$. Fluid Mech. 22, 103.

2. UCHIDA, T. et al., 1967 Ann. Rev。 Inst. of Plasma Physics, Nagoya (Japan) 20 .

3. LELOUP, C. and TAUSSING, R.T. 1968 ibid.

4. TANIUTI, T and WEI, C. 1968 J. Phys. Soc. Japan 24, 4, 941.

5. HAMADA, S., KAWAKAMI, I. and SATO, M. $1968 \mathrm{~J}$. Phys. Soc. Japan 25, 2, 542 .

6. CEESTER, W. 1960 Advances in Applied Mechanies Vol. VI, Accademic Press Inc. New York. 
付 録

ここで注本文の(5)式をそのをつ扱らことにょり我々の方法と通常の特性曲線の方法との関係を示

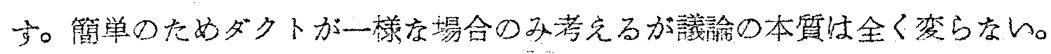

「に逆があるもされば(5)式は

$$
\mathrm{U}_{\mathrm{t}}+\mathrm{A}^{\prime} \mathrm{U}_{\mathrm{x}}=0
$$

と暂け为 $\left(\mathrm{A}^{\prime} \equiv \mathrm{I}^{-1} \mathrm{~A}\right)$ 。要ず

$$
1 A^{\prime}=\lambda 1
$$

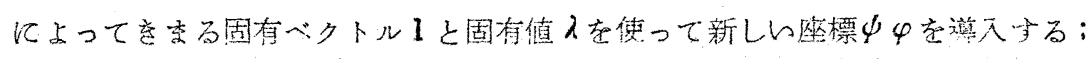

$$
\begin{aligned}
& \psi: \frac{\partial \psi}{\partial \mathrm{t}}+\lambda \frac{\partial \psi}{\partial \mathrm{x}}=0 \\
& \varphi: \varphi=\mathrm{t}
\end{aligned}
$$

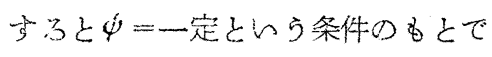

$$
\begin{gathered}
\int 1 \mathrm{~d} U=\mathrm{c}(\psi) \\
\frac{\mathrm{d} x}{\mathrm{dt}}=-\frac{\psi_{\mathrm{t}}}{\psi_{\mathrm{x}}}=\lambda
\end{gathered}
$$

が成立与る。(A・1) 式から波の速度として

$$
\lambda=\left\{\begin{array}{l}
\lambda_{+} \equiv \mathrm{u}+\mathrm{a} \\
\lambda=\mathrm{u}-\mathrm{a}
\end{array}\right.
$$

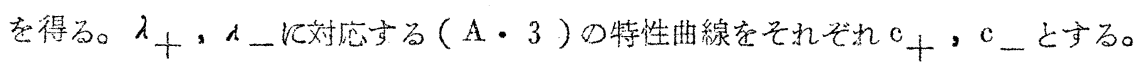

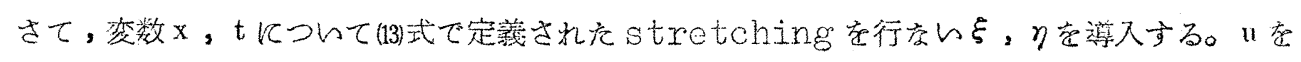
a。のまわりで展開すると

$$
\mathrm{u}=\mathrm{a}_{0}+\epsilon \mathrm{u}_{1}+\cdots
$$

とれから $(A \cdot 2)$ 式壮 
Reductive Perturbation Method in Nonlinear Wave Propagation III. Formation and Developement of Shock Waves in a Non-uniform Duct

$$
\begin{aligned}
& \epsilon \frac{d \xi}{d \eta}=2 a_{0}+\epsilon u_{1} \cdots c_{+}^{c_{+}} \\
& \epsilon \frac{d \xi}{d \eta}=\epsilon u_{1} \quad \cdots \cdots
\end{aligned}
$$

となるししがっで+は

$$
\frac{\mathrm{d} \xi}{\mathrm{d} \eta} \approx \frac{2 \mathrm{a}_{0}}{\epsilon}
$$

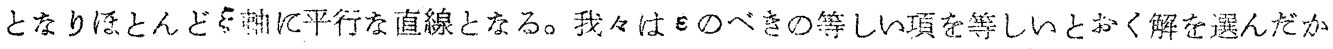

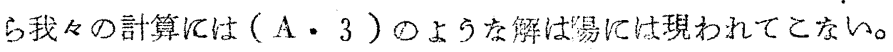

